\title{
Comment lutter contre la désinformation scientifique? Journée Sciences et Médias 2018
}

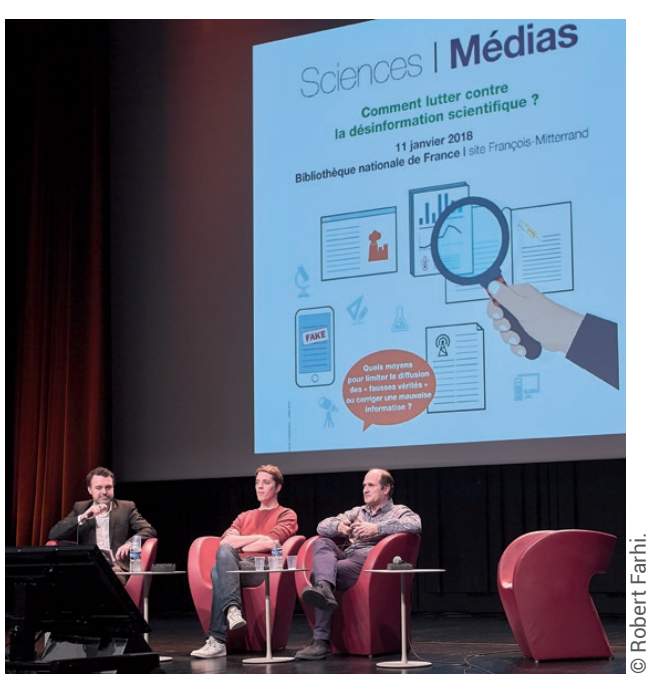

De gauche à droite : Daniel Fiévet (animateur de la journée), Gary Dagorn et Jean-Marc Bonmatin.

300 personnes présentes simultanément dans l'auditorium.

Les sciences ont, depuis très longtemps, fait l'objet de désinformations ou, pour le moins, de doutes de la part de l'opinion publique. Un récent sondage de l'Ifop [1], commenté en ouverture de la journée par Daniel Hennequin, président de la commission Culture scientifique de la SFP, montre que 9\% d'un échantillon représentatif de la population française est prête à adhérer à la thèse selon laquelle la Terre serait plate et non pas ronde.

Mais c'est le réchauffement climatique qui a fait l'objet, ces dernières années, de la campagne de désinformation la plus intense et la plus médiatisée. Comme l'a souligné, dans sa conférence introductive, Valérie Masson-Delmotte, coprésidente du premier groupe de travail (" éléments scientifiques ») du Groupe d'experts intergouvernemental sur l'évolution du climat (GIEC), dès les années 2009-2010, les actuel. La journée fut d'ailleurs un suc le pic de fréquentation s'étant situé à
À l'heure où l'exécutif français se propose de légiférer sur ces "fausses informations " ("fake news") et où celles-ci sont sans cesse dénoncées par la présidence américaine, le sujet abordé lors de cette quatrième journée Sciences et Médias ne pouvait être plus
L'information objective et scientifique pour lutter contre la désinformation est déficiente en France. Il n'existe pas, non plus, de coordination ni face à une éventuelle campagne massive de désinformation.

La précédente édition de Sciences et
Médias, en 2016, s'était interrogée sur les moyens à mettre en place pour parler de science aux jeunes. Dans une logique de sur la question de l'information scientifique dans les médias et son utilisation. En effet, technologies d'information et de communication a fortement modifié notre façon l'information. Elle est devenue instantanée et prend des formes très diverses. Au sein de cette variété, de nombreux travers laissent place à la surinterprétation, voire à la diffusion de "fausses vérités".

\author{
Le 11 janvier 2018 s'est tenue à la Bibliothèque nationale \\ de France (quai François Mauriac, Paris, $13^{\mathrm{e}}$ ) la quatrième journée \\ Sciences et Médias. Le but de ces journées est de participer \\ à la réflexion sur la façon dont les médias se saisissent des questions \\ scientifiques. L'édition 2018 était organisée par l'Association \\ des Journalistes Scientifiques de la Presse d'Information (AJSPI), \\ la Bibliothèque nationale de France (BnF), la Société Chimique \\ de France (SCF), la Société Française de Physique (SFP), la Société \\ Française de Statistique (SFdS), la Société Informatique de France \\ (SIF), la Société de Mathématiques Appliquées et Industrielles \\ (SMAI) et la Société Mathématique de France (SMF).
}

- . . . 
opposants à la thèse du réchauffement climatique n'ont eu de cesse de manipuler le doute quant à la réalité de l'origine anthropique du réchauffement. Le doute est inhérent à la démarche scientifique, et il est alors très facile de l'utiliser comme argument premier de la négation des faits. Le changement climatique demande une vision d'ensemble et un certain recul. Contrairement à une idée fortement ancrée dans l'esprit du public, il est disjoint des prévisions météorologiques. Malheureusement, le fil des médias ne donne qu'une vision parcellaire de certains éléments, met en lumière des faits extraits de leur contexte, comme le serait un détail d'une toile impressionniste. Il est facile aux « marchands de doute " de profiter de la complexité de la problématique pour dénigrer les outils et modèles climatologiques, et par voie de conséquence la responsabilité humaine du réchauffement climatique.

En outre, la participation au débat, non seulement de scientifiques, mais aussi de politiques, d'entreprises, d'ONG, tous animés de convictions intimes différentes, ne permet pas au public de se faire une idée précise et objective de la situation.

L'information très rapidement diffusée, et de façon plus accessible à chacun, par l'intermédiaire des blogs et réseaux sociaux, supplante les articles scientifiques et donne l'illusion au public, notamment jeune, de comprendre la totalité des faits. C'est ainsi que la différence entre les publications scientifiques et les opinions émises par des pseudo-experts n'est plus perçue.

Le doute sur la qualité des articles scientifiques est renforcé par la présence croissante de journaux prédateurs, et la communication est souvent biaisée, y compris par les grandes publications scientifiques à la recherche de scoops.

Face à cette situation, la réponse de la communauté scientifique française doit être plus adaptée et structurée. Contrairement à ce qui se passe dans les pays anglo-saxons, l'information objective et scientifique pour lutter contre la désinformation est déficiente en France. Il faut informer le grand public par une vulgarisation bien faite, par une éducation sur ce que sont la démarche scientifique et le rôle des véritables experts, et par un accès facilité aux données scientifiques. Il n'existe pas, non plus, de coordination ni de préparation du monde scientifique face à une éventuelle campagne massive de désinformation.
Enfin, un effort doit être conduit dans le secteur de l'éducation : les élèves du primaire et du collège ne sont pas assez sensibilisés à ce qu'est la démarche scientifique, et leurs enseignants, notamment ceux du primaire, doivent être rassurés et armés pour répondre aux questionnements scientifiques des élèves et aux tentatives de désinformation.

La table ronde qui suivait, animée par Daniel Fiévet (France Inter, La Tête au Carré), maître de cérémonie de la journée, avait pour thème "Comment choisit-on les experts scientifiques pour les médias ? " et rassemblait trois responsables de médias : Audrey Mikaëlian, Julien Guillaume et Mickaëlle Bensoussan.

Audrey Mikaëlian, journaliste de télévision, insiste sur la nécessaire compétence des experts, mais aussi et surtout sur leurs capacités à s'exprimer clairement et à se mettre à la portée du public. Avant d'aborder, par exemple, comment la sélection des reproducteurs bovins, puis le génie génétique, ont permis à l'homme de " fabriquer " des vaches parfaitement adaptées à la production et la consommation intensives, il est nécessaire que le public sache qu'une vache se trait deux fois par jour !

Le service de presse du CNRS, dont Julien Guillaume est responsable, a constitué une base d'experts dans tous les domaines, et en particulier les sciences humaines et sociales (SHS), qui font l'objet du plus grand nombre de demandes; cette base d'experts permet ainsi de répondre aux sollicitations, quelquefois très urgentes, des médias, en fournissant plusieurs noms sur un thème donné.

Mickaëlle Bensoussan, rédactrice en chef de Ça m'intéresse, interroge très souvent, surtout dans des domaines polémiques (tels que la santé), plusieurs experts susceptibles de donner des éclairages différents d'une même problématique. Prendre parti reviendrait en effet à occulter un ou plusieurs aspects d'une question.

L'apparition récurrente des mêmes chercheurs dans les médias relève de mécanismes très simples : ce sont ceux qui sont les plus disponibles et les plus intéressés à communiquer qui réagissent le plus vite et répondent au téléphone! Les journalistes s'échangent les bases de données, et font souvent appel aux chercheurs avec qui le partage a été le plus fructueux dans des expériences précédentes.
Les trois intervenants de la table ronde ont évoqué les réticences de certains scientifiques à répondre à leurs sollicitations. Ces derniers ont très souvent peur de voir leurs propos déformés, et pensent, à tort, qu'une intervention dans les médias ne leur rapporterait rien. Enfin, les femmes semblent plus réservées pour répondre aux sollicitations des médias. Elles demandent souvent à réfléchir, ce qui de fait les exclut compte tenu de l'immédiateté de certains moyens de diffusion.

La conclusion de cette table ronde aura été unanime : il n'y a pas assez de journalistes scientifiques!

Quel est le rôle des scientifiques dans la désinformation ? Ce sujet faisait l'objet de deux conférences.

Nicolas Chevassus-au-Louis, journaliste à Mediapart, a démonté les mécanismes de la désinformation, qui s'appuient sur le négationnisme (au sens large de négation des faits), la théorie du complot et l'ignorance. La première étape consiste à instiller le doute: "Tu ne trouves pas ça bizarre, toi ?", et à chercher "à qui profite le crime " (Cui prodest). Les points clefs de la théorie du complot des attentats du 11 septembre peuvent être rapprochés des thèses négationnistes produites par Robert Faurisson [2], ou, dans une bien moindre mesure, de l'ouvrage publié par Claude Allègre en 2010 (L'imposture climatique).

La seconde étape est de proposer des versions alternatives, puis, troisième étape, de les faire défendre par des scientifiques de renom ou des témoins "dignes de foi ", tels qu'un ancien rescapé des camps d'extermination (argument d'autorité). Y participent également des revues d'apparence scientifique, mais qui ne résistent pas à l'analyse de véritables experts du domaine. On peut citer des exemples, comme le Journal of Historical Review, qui véhicule des thèses négationnistes, ou le Journal of 9/11 studies, mais aussi des revues prédatrices. C'est ainsi qu'on peut trouver, dans The Open Chemical Physics Journal, un article démontrant la présence de traces d'explosifs dans les restes des tours jumelles du World Trade Center.

Il est donc malheureusement toujours possible de trouver des scientifiques ou des revues spécialisées vecteurs de la négation et de la désinformation.

Dans un monde où les journalistes perdent peu à peu le contrôle de la diffusion des informations et où elles sont toutes 


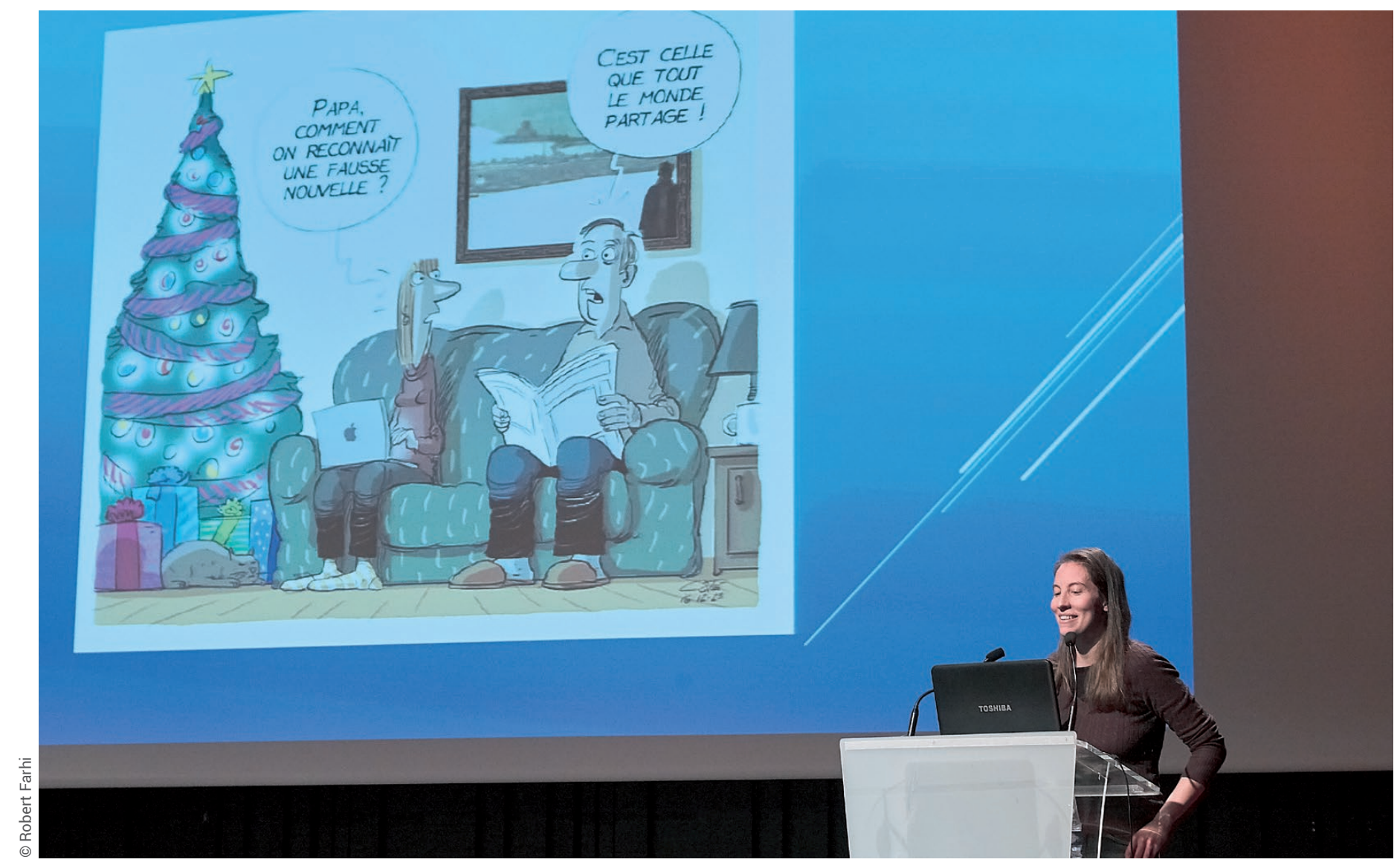

Conférence de Francesca Musiani.

mises sur un pied d'égalité par le biais des réseaux sociaux et d'Internet (Google, Facebook...), il devient de plus en plus nécessaire de redonner la parole aux scientifiques et de fournir au public une information digne de foi, vérifiée et contrôlée par une expertise rigoureuse.

C'est ce que fait Emmanuel Vincent (Université de Californie), avec son initiative Climate Feedback [3]. Elle repose sur un réseau international de scientifiques qui trient les faits et les séparent de la fiction dans le domaine du changement climatique, afin de permettre au lecteur de savoir quelle information est crédible. Ces spécialistes sont mis à contribution, sur la base du volontariat, pour juger, commenter, et noter, dans l'ensemble des médias, les publications dans le domaine de la climatologie et du changement climatique.

Les principaux biais relevés sont le cherrypicking, qui consiste à extraire un petit fait ou une donnée secondaire pour la mettre en avant, les conflits d'intérêt (notamment dans les domaines médicaux et pharmaceutiques), les raisons religieuses, économiques ou politiques qui conduisent à produire de la désinformation, la notoriété mal placée des intervenants dans le débat, la construction de blogs véhiculant de fausses informations sans contrôle de l'hébergeur. Il est très facile d'instiller le doute dans l'esprit du lecteur, mais ô combien plus difficile de lutter contre la désinformation. Les moyens à mobiliser sont considérables.

L'intervention de scientifiques compétents et pédagogues dans les médias est primordiale pour lutter contre la désinformation. Encore faut-il que ces scientifiques y soient préparés. Ce sujet faisait l'objet d'une seconde table ronde, "Comment préparer les scientifiques aux médias?", à laquelle participaient Cécile Michaut, journaliste scientifique et formatrice en media training, Audrey Mikaëlian, et Roberto Vargiolu, ingénieur de recherche au CNRS et vulgarisateur scientifique.

Il n'est pas donné à tous les scientifiques, fussent-ils compétents, d'expliquer ce qu'est le boson de Higgs en 20 secondes... Dans toute intervention, comme dit plus haut, il faut considérer qu'on s'adresse à un public dont on ne connaît pas le niveau. Il faut donc expliquer pourquoi c'est intéressant, raconter une histoire, simplifier le vocabulaire, ne pas utiliser de mots à double sens (comme le mot "modèle " qui possède pour le grand public un sens très différent de celui que lui donne le scientifique), ne jamais entrer dans les détails, partir de très bas pour conduire le lecteur ou l'auditeur le plus haut possible. Les surfaces doivent se compter en terrains de football, les volumes en piscines ou en dés à coudre !

Savoir quel message faire passer et comment le faire s'apprend. Une intervention dans les médias est une discussion entre un journaliste et un chercheur, et l'entretien est avant tout un partage, basé sur une générosité commune en direction du public. Le journaliste doit, de son côté, rester ouvert, savoir quitter le chemin qu'il s'était tracé lorsque nécessaire, et ne pas trop préparer son entretien. Il n'est en aucun cas le porte-parole du scientifique.

Lorsque le chercheur ne s'estime pas compétent ni suffisamment sûr de lui, il doit savoir décliner la proposition d'entretien. À l'inverse, lorsqu'il l'accepte, il sait qu'il n'aura aucun contrôle de ce qui sera écrit ou diffusé. Il n'aura jamais que le 
contrôle de ce qu'il aura dit. Il n'existe en effet aucun droit de relecture, et il appartient au seul journaliste de ne pas trahir les mots ou le message prononcés par le scientifique.

L'après-midi débutait sur une troisième table ronde, intitulée "Médias numériques : comment sont créées et vérifiées les informations ? ". Elle rassemblait Pierre Kerner (Café des Sciences), Florence Porcel, créatrice d'une chaîne YouTube (La Folle Histoire de l'Univers), et Didier Pourquery, directeur de rédaction de The Conversation France. Ces médias, de natures différentes, sont cependant tous confrontés au problème du contrôle de l'information.

Un blog, tel que celui du Café des Sciences, permet toujours la publication d'un correctif lorsqu'une erreur est découverte. La participation active de scientifiques travaillant en réseau permet un travail rigoureux de vérification de l'information.

Tel n'est pas le cas des vidéos publiées sur YouTube, dont le contenu n'est évidemment pas vérifié par l'hébergeur. Le contrôle de l'information n'appartient alors qu'à l'auteur de la vidéo. Florence Porcel s'appuie sur une collaboration avec des scientifiques, qui lui permet de vérifier l'information qu'elle diffuse, et mentionne toujours, au bas de ses vidéos, les sources utilisées.

The Conversation France rédige, en langue française, une lettre électronique quotidienne et des articles destinés à la presse écrite, dans tous les domaines, y compris scientifiques. Les auteurs des articles sont des chercheurs et des universitaires. La vérification de l'information est d'autant plus cruciale que le rythme de parution est soutenu, mais le travail en réseau permet de répondre à cette nécessité.

On peut déplorer la publication d'articles, de revues, de vidéos, de blogs aux titres racoleurs, dont le sérieux peut être mis en doute et ne peut être évalué qu'en allant vérifier le contenu scientifique et les sources. On pourrait, certes, envisager la création de "labels de qualité " de l'information, mais qui risqueraient de se révéler à double tranchant. Quel que soit le média, il va de soi que tous ses contributeurs doivent être attentifs à la véracité de l'information diffusée. Mais dans le cas des médias numériques, dont l'interactivité est la caractéristique première, la confiance réciproque, la transparence, la discussion et le travail en réseau sont les conditions premières d'une bonne qualité de l'information. Le choix des experts, sur la base de leur domaine de recherche, est aussi une garantie.

Deux conférences portaient sur les algorithmes : "Comment les algorithmes font-ils remonter des informations et permettent-ils de traquer la désinformation?"

La neutralité du Net a fait récemment l'objet de nombreux débats, principalement aux États-Unis. Cependant, l'objectivité de l'information obtenue sur Internet par le grand public fait déjà partie du passé. Alors qu'une recherche réalisée à l'aide d'un moteur, tel que Google, par exemple, faisait apparaitre autrefois un classement de l'information en fonction de la popularité de la référence, il n'en est plus de même aujourd'hui. Les algorithmes utilisés par les moteurs de recherche retournent une information ciblée de façon individuelle, en fonction de l'historique des requêtes de l'internaute et de son profil. Cette nouvelle personnalisation, basée sur des considérations essentiellement publicitaires et commerciales, conduit, nous dit Francesca Musiani (Institut des sciences de la communication, CNRS), à une propagande individualisée qui n'est ni plus ni moins qu'un biais de l'information : on assiste ainsi à un enfermement idéologique de l'internaute, qui ne trouve, comme réponses à ses requêtes, que des informations qui confortent ses convictions. Les rumeurs sont ainsi propagées, puis industrialisées, car générant des revenus.

Dans ce contexte, on ne sait plus différencier le vrai du faux, ni qui est à l'origine d'une fake news. Cette privatisation de la gouvernance est une véritable menace à la liberté d'expression, mais est aussi source de désinformation.

Comment lutter contre cette tendance ? La réponse n'est pas simple. Il faudrait modifier les algorithmes de recommandation, créer des entraves aux revenus publicitaires, censurer les profils, faire œuvre de pédagogie auprès du grand public. Cette démarche ne peut être que concertée et impulsée par les différentes parties prenantes elles-mêmes.

"Si l'on sait exactement ce qu'on va faire, à quoi bon le faire ? " C'est par cette citation que Gilles Dowek, chercheur à l'Inria, sème le doute sur la véracité de l'information, au-delà de la seule informa- tion scientifique. Cette citation, attribuée à Picasso, ne se trouve que dans les Conversations avec Christian Zervos, publiées en 1935 dans la revue Cahiers d'Art. Picasso faisait exclusivement référence à son approche de la peinture, selon laquelle l'artiste ne doit jamais connaitre d'avance le résultat d'une œuvre commencée. Cette citation a cependant été abondamment utilisée dans d'autres contextes.

Pire encore, la phrase, attribuée à Voltaire, "Je ne suis pas d'accord avec ce que vous dites, mais je me battrai jusqu'à la mort pour que vous ayez le droit de le dire ", a été écrite par Evelyn Beatrice Hall, dans un ouvrage publié en 1906, pour résumer sa pensée, mais n'a jamais été écrite par le philosophe. Et pourtant, cette phrase apocryphe est le plus souvent attribuée à Voltaire lui-même.

Ces deux exemples montrent combien l'information peut être biaisée ou même créée de toutes pièces. C'est certes sur Internet et la toile qu'il est possible de trouver le plus souvent de fausses informations, mais les médias classiques n'échappent pas à la règle, comme le montrent les deux citations précédentes.

Au-delà de la coupable désinformation, la manipulation des faits est un véritable péché, bien plus grave. L'exemple cité par Gilles Dowek illustre parfaitement cette technique : les défenseurs de la chasteté nient l'efficacité du préservatif masculin, puisque c'est dans les pays d'Afrique, où les États-Unis envoient le plus de préservatifs, que l'épidémie de SIDA fait le plus de ravages !!

Il n'existe malheureusement pas d'algorithme permettant de vérifier la véracité des faits ou des informations. On pourrait en imaginer un permettant de détecter une logique défaillante, comme dans le cas précédent, mais il n'existe pas encore.

La seule façon, aujourd'hui, de détecter une fausse information est d'analyser les arguments avancés. On trouve encore malheureusement trop souvent des arguments du type "Tout le monde dit " ou, pire, "Il y a un expert qui dit ». Il faut bien insister sur le fait que la vérité scientifique n'est pas une opinion.

Les deux dernières conférences de la journée abordaient « la question de l'attitude à adopter face à une information erronée".

Jean-Marc Bonmatin, du centre de biophysique moléculaire du CNRS, se 
trouve au cœur du débat sur les néonicotinoïdes. Ces substances couvrent à elles seules 40\% des besoins français en pesticides. Elles agissent sur le système nerveux central des insectes, provoquant la paralysie et la mort. Le débat oppose les apiculteurs d'une part, et les agriculteurs et industriels de l'agrochimie d'autre part, sur la responsabilité de ces substances dans la mortalité observée des abeilles. Outre les questions déontologiques et relatives à la biodiversité, les montants évoqués par les parties prenantes sont considérables. Le marché mondial des néonicotinoïdes est évalué à 2,5 milliards d'euros par an. Mais dans le même temps, la pollinisation génère 153 milliards d'euros.

Dans ce contexte, les contrevérités sont légion. Un journal canadien, Le Devoir, titrait par exemple, en septembre 2017 : «L'utilisation des néonicotinoïdes ne tue pas les abeilles ». Une lecture rigoureuse montre cependant que cet article relève plus d'une opinion que d'une analyse rigoureuse. De la même façon, une rumeur s'est répandue selon laquelle un rapport de l'Union européenne évaluait à 4,7 milliards d'euros les pertes liées à un éventuel abandon des néonicotinoïdes. Or, ce rapport n'a jamais existé ! Enfin, Jean-Marc Bonmatin a dû corriger, en argumentant à de nombreuses reprises, l'article sur les néonicotinoïdes dans Wikipédia qui, rappelle-t-il, constitue la source première d'information scientifique de $90 \%$ des jeunes.

Comment le chercheur doit-il se positionner sur un sujet aussi brûlant et lourd de conséquences?

En premier lieu, il doit analyser les faits et les travaux, leurs sources, et mettre au jour les éventuels conflits d'intérêts. Il faut rappeler que les grands groupes industriels ont mis en place des stratégies visant à instaurer le doute (tabac, amiante, énergies fossiles...) et qu'ils financent de nombreux projets de recherche, voire des experts... Dans un second temps, il doit communiquer avec rigueur et clarté, en argumentant face aux contrevérités. Enfin, son devoir est d'alimenter le débat contradictoire par des faits scientifiques solides et de s'impliquer dans l'expertise.

Le dernier intervenant de la journée était Gary Dagorn, journaliste aux Décodeurs, une rubrique du site Internet du journal Le Monde. Compte tenu de l'ingérence de la science dans les débats de société actuels, tels que les vaccins ou le cancer, sa part dans les analyses fournies par Les décodeurs est passée à $4 \%$, en augmentation significative au cours de ces dernières années.

Le partage des informations via les réseaux sociaux constitue une véritable caisse de résonance à la désinformation : plus les informations sont grosses et peu crédibles, et plus elles sont cependant partagées. Il convient donc de remonter à la source. Le Monde travaille par exemple avec Facebook pour pouvoir mieux appréhender sa démarche et lutter contre l'apparition des fausses informations.

Enfin, un gros travail de pédagogie est nécessaire à destination du public. Le moteur de recherche "Décodex " du quotidien Le Monde, qui permet de vérifier si la source de l'information (page Web ou site Internet) est plutôt fiable ou non, est, de ce point de vue, un outil efficace mis à la disposition des lecteurs.

Mais Gary Dagorn se pose tout de même la question de savoir si, dans un tel contexte, "décoder " des contrevérités ne produit pas l'effet inverse, à savoir celui de les amplifier...

Patricia Pineau, rédactrice en chef de L'Actualité Chimique, appelée à faire une synthèse de la journée, a conclu en remarquant que l'assistance était plutôt jeune, et que la nouvelle génération n'hésitait pas à s'impliquer dans la diffusion de la vérité scientifique et dans la lutte contre la désinformation.

\section{En conclusion}

S'il est très facile de créer de la désinformation et des rumeurs, puis de les véhiculer, il est beaucoup plus difficile de les démentir. Il faut tout d'abord travailler à la source. Les médias peuvent jouer un rôle crucial dans la lutte contre les fausses informations, et beaucoup d'entre eux le font déjà. Plus récemment, le 19 janvier 2018, Mark Zuckerberg, CEO de Facebook, a déclaré sur sa page vouloir faire de 2018 l'année de la lutte contre les contenus problématiques. Pour y parvenir sans pour autant être accusé de censure, il serait demandé aux utilisateurs leur avis sur leurs sources et la confiance qu'ils leur accordent. Les algorithmes seraient modifiés en conséquence pour tenir compte de ces retours et hiérarchiser les sources d'information. L'outil Décodex, évoqué plus haut, œuvre dans le même sens, et un partenariat entre Facebook et plusieurs médias français [4] existe depuis février 2017.

Il est tout aussi important d'éduquer le public à faire la différence entre le vrai et le faux, à faire le tri des informations glanées sur le Net et les réseaux sociaux, bref, à différencier l'information du savoir. Encore faut-il pour cela que le référencement des sites et des informations ne soit pas soumis à des biais, d'origine publicitaire ou autre.

Enfin, le rôle des chercheurs et universitaires dans cette lutte est triple. Tout d'abord, analyser les sources et leur qualité, la véracité de l'information, et détecter d'éventuels conflits d'intérêts. Ensuite, contribuer à l'information, en s'impliquant auprès des médias, à la condition d'être à la fois compétent dans le domaine et de tenir un langage clair et accessible au grand public. Enfin, construire des réseaux, à l'instar de ce qui existe dans les pays anglo-saxons, afin de coordonner leurs réponses et faire face à une éventuelle campagne de désinformation massive.

$\mathrm{La}$ tâche est donc difficile, mais pas impossible. Comme le dit Patricia Pineau dans ses mots de conclusion de la journée, il convient de rester optimiste !

Robert Farhi Commission Culture scientifique de la SFP

Site des journées Sciences et Médias http://sciencesetmedia.org/index.php

Vidéos en ligne des interventions

de la journée

www.youtube.com/channel/ UCFTd8PVVJvw51SyQmdCM7xA

\section{Références}

1 Ifop pour la Fondation Jean Jaurès et Conspiracy Watch, « Enquête sur le complotisme " (décembre 2017), p. 67.

2. Le Monde, 29 décembre 1978

"Le "problème des chambres à gaz" ou la rumeur d'Auschwitz ".

3. https://climatefeedback.org

4. Agence France-Presse (AFP), BFMTV, France Télévisions, France Médias Monde, L'Express, Le Monde, Libération et 20 Minutes. 